Bull. Austral. Math. Soc.

VOL. 49 (1994) [413-419]

\title{
DERIVED MENDELSOHN TRIPLE SYSTEMS
}

\section{ZORAN STOJAKOVIĆ}

\begin{abstract}
Mendelsohn triple system of order $v$ which can be extended to a tetrahedral quadruple system of order $v+1$ we call a derived Mendelsohn triple system. We consider some properties of derived Mendelsohn triple systems and give some results on their existence.
\end{abstract}

\section{INTRODUCTION}

Mendelsohn triple systems (MTSs), which represent a generalisation of Steiner triple systems, were introduced in [6]. A MTS of order $v$ is a pair $(S, T)$, where $S$ is a finite set of $v$ elements and $T$ is a collection of cyclic triples $\langle a b c\rangle=\{(a, b),(b, c),(c, a)\}$, $a, b, c$ distinct elements from $S$, such that every ordered pair of distinct elements from $S$ belongs to exactly one cyclic triple from $S$. By $\operatorname{MTS}(v)$ we denote a MTS of order $v$.

In [14] a class of quadruple systems called tetrahedral quadruple systems (TQSs) was defined. TQSs represent a generalisation of Mendelsohn triple systems different from generalisations in $[11,13]$. A TQS of order $v$ is a pair $(S, T)$, where $S$ is a finite set of $v$ elements and $T$ is a family of directed quadruples $\langle a b c d\rangle, a, b, c, d$ distinct elements of $S$, such that every ordered triple of distinct elements of $S$ belongs to exactly one directed quadruple from $T$. A directed quadruple $\langle a b c d\rangle$ is the following set of 12 ordered triples

$$
\begin{aligned}
\langle a b c d\rangle=\{(a b c),(b c a),(c a b),(a d b),(d b a),(b a d) \\
\\
(a c d),(c d a),(d a c),(b d c),(d c b),(c b d)\} .
\end{aligned}
$$

By TQS $(v)$ we denote a TQS of order $v$.

It was proved in [14] that TQSs are equivalent to generalised idempotent alternating symmetric (GIAS) 3-quasigroups, their properties were investigated and some parts of the spectrum of TQSs determined. In [3] further investigation of TQSs was carried on and it was proved that the spectrum of TQSs consists of all $v$ such that $v \equiv 1,2,4,5,8,10(\bmod 12)$.

Received 16th June, 1993

This research was supported by Science Fund of Serbia.

Copyright Clearance Centre, Inc. Serial-fee code: 0004-9729/94 \$A2.00+0.00. 
The sequence $x_{m}, x_{m+1}, \ldots, x_{n}$ is denoted by $\left\{x_{i}\right\}_{i=m}^{n}$ or by $x_{m}^{n}$. If $m>n$, then $x_{m}^{n}$ will be considered empty.

An $n$-ary groupoid ( $n$-groupoid) $(Q, f)$ is called an $n$-quasigroup if the equation $f\left(a_{1}^{i-1}, x, a_{i+1}^{n}\right)=b$ has a unique solution $x$ for every $a_{1}^{n}, b \in Q$ and every $i \in\{1, \ldots, n\}$.

By $S_{n}$ we denote the symmetric group of degree $n$ and by $A_{n}$ its alternating subgroup.

\section{Alternating SYMmetric 3-QUASIGROUPS AND TQSS}

In $[12,14]$ a class of $n$-groupoids called alternating symmetric $n$-groupoids was defined and considered.

Definition 1.: A 3-groupoid $(S, f)$ is alternating symmetric (AS) if for every permutation $\sigma \in A_{4}$ and all $x_{1}^{4} \in S$

$$
f\left(\left\{x_{\sigma(i)}\right\}_{i=1}^{3}\right)=x_{\sigma(4)} \Longleftrightarrow f\left(x_{1}^{3}\right)=x_{4} .
$$

This definition of AS-3-groupoids can be given also in another equivalent form.

THEOREM 1. [14] A 3-groupoid $(S, f)$ is $A S$ if and only if the following identities are satisfied:

$$
\left\{\begin{array}{l}
f(x, y, z)=f(y, z, x) \\
f(y, f(x, y, z), z)=x
\end{array}\right.
$$

Every AS-3-groupoid is necessarily a 3-quasigroup.

A 3-groupoid $(S, f)$ is called generalised idempotent (GI) if and only if for all $x, y \in S$

$$
f(x, y, y)=f(y, x, y)=f(y, y, x)=x .
$$

An AS-3-groupoid which is GI is called a GIAS-3-groupoid.

So, a 3-groupoid $(Q, f)$ is a GIAS-3-groupoid if and only if it satisfies the following identities:

$$
\left\{\begin{array}{l}
f(x, y, z)=f(y, z, x) \\
f(y, f(x, y, z), z)=x \\
f(x, y, y)=x
\end{array}\right.
$$

Hence the class of all GIAS-3-groupoids is a variety.

In [14] it is proved that finite GIAS-3-groupoids are equivalent to TQSs.

THEOREM 2. [14] Every TQS of order $v$ defines and is defined by a GIAS-3groupoid of order $v$. 
If $(S, T)$ is a TQS of order $v$, and $f$ is defined for distinct elements $x, y, z, u \in S$ by

$$
f(x, y, z)=u \Longleftrightarrow\langle x y z u\rangle \in T
$$

and

$$
f(x, y, y)=f(y, x, y)=f(y, y, x)=x,
$$

then $(S, f)$ is GIAS-3-groupoid of order $v$. Conversely, if $(S, f)$ is a GIAS-3-groupoid of order $v$, then by (1) a TQS $(S, T)$ of order $v$ is defined.

Since Steiner quadruple systems are equivalent to generalised idempotent totally symmetric (GITS) 3-quasigroups (the definition of a totally symmetric 3-quasigroup is obtained if in Definition 1 we replace $A_{4}$ by $S_{4}$ ), and every GITS-3-quasigroup is a GIAS-3-quasigroup, we see that TQS represent a generalisation of Steiner quadruple systems. Some questions concerning the algebraic theory of Steiner quadruple systems were considered in $[2,5,8,9,10]$.

\section{DERIVED MTSS}

If $(S, T)$ is a TQS of order $v$ and $x$ is any element in $S$, we shall denote $S \backslash\{x\}$ by $S_{x}$ and the set of all cyclic triples $\langle a b c\rangle$ such that $\langle x a b c\rangle \in T$ by $T(x)$. Then $\left(S_{x}, T(x)\right)$ must be a MTS of order $v-1$, which we call a derived MTS (briefly DMTS) of the TQS $(S, T)$.

The DMTSs are equivalent to retracts of GIAS-3-quasigroups. If $(S, f)$ is a GIAS3-quasigroup, $a \in S$ a fixed element, then by

$$
x y=f(a, x, y)
$$

a retract $(S, \cdot)$ of $(S, f)$ is defined. Since $(S, f)$ is a 3-quasigroup, $(S, \cdot)$ is a binary quasigroup. But $(S, f)$ is also $\mathrm{AS}$, hence

and

$$
\begin{aligned}
& x y=f(a, x, y)=f(x, y, a)=f(y, a, x) \\
& x y=z \Leftrightarrow f(a, x, y)=z \Leftrightarrow f(a, z, x)=y \Leftrightarrow z x=y .
\end{aligned}
$$

A quasigroup satisfying the equivalence $x y=z \Leftrightarrow z x=y$ (or the equivalent identity $(x y) x=y)$ is called semisymmetric, hence $(S, \cdot)$ is a semisymmetric quasigroup.

Further, from $f(a, a, x)=f(a, x, a)=x$ and $f(a, x, x)=a$, we get that for all $x \in S, a x=x a=x, \quad x^{2}=a$, that is, $(S, \cdot)$ is a semisymmetric unipotent loop with the unit $a$. Semisymmetric unipotent loops we shall call M-loops. M-loops which can be obtained as retracts of GIAS-3-quasigroups will be called derived M-loops. 
M-loops were considered in [7] where it was proved that every M-loop of order $v$ defines a $\operatorname{MTS}(v-1)$ (and also that every such loop can be defined by one identity $x(((y y) z) x)=z)$. As we have seen, derived M-loops of order $v$ are equivalent to DMTSs of order $v-1$. If $(S, \cdot)$ is a derived M-loop with the unit $a$, and $(S, f)$ is a GIAS-3-quasigroup such that $x y=f(b, x, y)$ for some $b \in S$, then it follows that $a=b$.

An interesting problem about Steiner triple systems (which is far from solved) is whether or not every Steiner triple system is derived of some Steiner quadruple system. The similar question for MTSs - is every MTS derived of some TQS - has a negative answer. Since the spectrum of MTSs consists of all $v \equiv 0,1(\bmod 3), v \neq 6$ and the spectrum of TQSs consists of all $v \equiv 1,2,4,5,8,10(\bmod 12)$, we get that no MTS of order $v \equiv 6,10(\bmod 12)$ is derived.

If we assume that two MTSs derived from a TQS for distinct elements $a, b \in Q$ have a triple $\langle x y z\rangle$ in common, then $f(a, x, y)=f(b, x, y)$, hence $a=b$, which is a contradiction. So, for different elements $a, b \in Q$ the two DMTSs must be disjoint.

Theorem 3. Let $(S, T)$ be a TQS $(v)$. If for every $a \in S$ a $D M T S\left(S_{a}, T(a)\right)$ is defined, then a family of disjoint MTSs is obtained such that $C(S)=\bigcup_{a \in S} T(a)$, where $C(S)$ is the set of all possible cyclic triples of elements from $S$.

PRoOF: We have already proved that for $a \neq b, T_{a} \cap T_{b}=\emptyset$, and by a simple calculation we get that every cyclic triple of elements from $S$ belongs to $\bigcup_{a \in S} T(a)$.

The following problem, which arises quite naturaly, was considered by several authors (see $[1,4]$ ). If $S$ is a set of $v$ elements, where $v$ is such that there exists a $\operatorname{MTS}(v)$, is it possible to partition $C(S)$ into $v-2$ subsets $T_{1}, \ldots, T_{v-2}$ such that each $\left(S, T_{1}\right), \ldots,\left(S, T_{v-2}\right)$ is a MTS? Such a collection of MTSs of order $v$ is called a large set of pairwiswe disjoint MTSs of order $v(\operatorname{LSMTS}(v))$ and so far only partial results on the existence of $\operatorname{LSMTS}(v)$ are known.

The family of MTSs obtained in Theorem 3 is not a $\operatorname{LSMTS}(v)$, but it is in some sense "large". In fact, it is a partition of $C(S)$ into $v$ MTSs of order $v-1$ and such a family we shall call quasi LSMTS(v) (QLSMTS(v)). So, the next theorem is valid.

THEOREM 4. For every $v \equiv 1,2,4,5,8,10(\bmod 12)$ there exists a $Q \operatorname{LSMTS}(v)$.

\section{Constructions of DMTs}

Theorem 5. If $\left(S_{1}, T_{1}\right)$ and $\left(S_{2}, T_{2}\right)$ are DMTSs of orders $v_{1}$ and $v_{2}$ respectively, then the MTS of order $v_{1} v_{2}+v_{1}+v_{2}$ equivalent to the direct product of $M$-loops defined by the given DMTs, is also derived.

Proof: Let $\left(S_{1}, T_{1}\right)$ and $\left(S_{2}, T_{2}\right)$ be DMTSs of orders $v_{1}$ and $v_{2}$ respectively. If $\left(\bar{S}_{1}, \cdot\right)$ and $\left(\bar{S}_{2}, *\right)$ are M-loops which are equivalent to the given DMTSs, where 
$\bar{S}_{1}=S_{1} \cup\{a\}, a \notin S_{1}, \quad \bar{S}_{2}=S_{2} \cup\{b\}, b \notin S_{2}$, then, since the class of M-loops is a variety, their direct product $\left(\bar{S}_{1} \times \bar{S}_{2}\right.$, o) is also an M-loop which defines a MTS of order $v_{1} v_{2}+v_{1}+v_{2}$.

M-loops $\left(\bar{S}_{1}, \cdot\right)$ and $\left(\bar{S}_{2}, *\right)$ are derived, hence there exist GIAS-3-quasigroups $\left(\bar{S}_{1}, f_{1}\right),\left(\bar{S}_{2}, f_{2}\right)$ such that $f_{1}(a, x, y)=x y, f_{2}(b, x, y)=x * y$. Since the class of GIAS3-quasigroups is a variety, the direct product of $\left(\bar{S}_{1}, f_{1}\right)$ and $\left(\bar{S}_{2}, f_{2}\right),\left(\bar{S}_{1} \times \bar{S}_{2}, y\right)$, is also a GIAS-3-quasigroup. If we define a retract of $g$ by $g((a, b), x, y)$, then this retract is in fact the direct product of M-loops $\left(\bar{S}_{1}, \cdot\right)$ and $\left(\bar{S}_{2}, *\right)$.

In the preceding theorem the assumption that $\left(S, T_{1}\right)$ and $\left(S, T_{2}\right)$ are DMTSs of orders $v_{1}$ and $v_{2}$, implies that $v_{1}, v_{2} \geq 3$. But since there exists an M-loop of order 2 (although there is no MTS which is equivalent to that M-loop, we can consider this M-loop to be equivalent to a DMTS(1) with empty set of triples), Theorem 5 can be extended to the case where $v_{1}=1$. Hence, as a consequence of Theorem 5 we get the next theorem which starting from a DMTS(v) gives a DMTS $(2 v+1)$.

Theorem 6. If $(S, T)$ is a $\operatorname{DMTS}(v)$, then there exists a $\operatorname{DMTS}(2 v+1)(R, K)$, such that $(S, T)$ is a subsystem of $(R, K)$.

A subsystem of a MTS $(R, K)$ is a MTS $(S, T)$ such that $S \subseteq R$ and $T \subseteq K$.

Theorem 7. Let $(R, K)$ be a $\operatorname{MTS}(2 v+1)$ having a $\operatorname{DMTS}(v)(S, T)$ as a subsystem. If the $M$-loop $(\bar{R}, \cdot)$ which is equivalent to $(R, K)$ is such that for every $a, b, c, d \in R \backslash S, a b=c d \Longrightarrow b a=d c$, then $(R, K)$ is itself derived.

Proof: Let $S=\left\{x_{1}, \ldots, x_{v}\right\}$ and let $A=R \backslash S=\left\{a_{1}, \ldots, a_{v+1}\right\}$. We form the following partition $\bar{A}^{2}=A_{1} \cup \ldots \cup A_{v}$ of the set of all ordered pairs of distinct elements of $A:(a, b) \in A_{i}$ if and only if $\left\langle x_{i}, a, b\right\rangle \in K$. In every set $A_{i}, i=1, \ldots, v$, each element of $A$ appears exactly once as the first and exactly once as the second component of an ordered pair. Also, if we replace every pair $(a, b)$ in $A_{i}$ by $(b, a)$ we get some class $A_{j}$.

Take an element $p \notin R$, let $S^{*}=S \cup\{p\}$ and $\left(S^{*}, T^{*}\right)$ be a TQS such that $\left(S_{p}^{*}, T^{*}(p)\right)=(S, T)$. Let $(A, B)$ be any TQS on the set $A$. If $y_{i}$ is the solution of the equation $a_{1} y_{i}=x_{i}, i=1, \ldots, v$, then $y_{i} \in A$, and we define a bijection $g: A \rightarrow S^{*}$ by $g\left(a_{1}\right)=p, g\left(y_{i}\right)=x_{i}, i=1, \ldots, v$. If in $A_{i}$ we replace every pair $(a, b)$ by $(g(a), g(b))$, we get a set which will be denoted by $S_{i}$. Then $S_{1} \cup \ldots \cup S_{v}$ is a partition of the set of all ordered pairs of distinct elements of $S^{*}$.

We define a set $K^{*}$ of directed quadruples on $R^{*}=S^{*} \cup A$ as follows. $T^{*} \cup B \subset K^{*}$ and if $a, b \in A, c, d \in S^{*}$, then $\langle a b c d\rangle \in K^{*}$ if and only if there exists $i \in\{1, \ldots, v\}$ such that $(a, b) \in A_{i},(c, d) \in S_{i}$.

We prove that $\left(R^{*}, K^{*}\right)$ is a TQS $(2 v+2)$. For every $i, \quad\left|A_{i}\right|=\left|S_{i}\right|=v+1$, hence we have formed a list of $v(v+1)^{2}$ directed quadruples of the form $\langle a b c d\rangle$, where 
$(a, b) \in A_{i},(c, d) \in S_{i}$, but since $\langle a b c d\rangle=\langle b a d c\rangle$ in that list every directed quadruple appears exactly twice, so $K^{*} \backslash\left\{T^{*} \cup B\right\}$ has $v(v+1)^{2} / 2$ elements. $\left|T^{*}\right|=|B|=$ $(v+1) v(v-1) / 12$, hence

$$
\left|K^{*}\right|=\frac{(v+1) v(v-1)}{6}+\frac{v(v+1)^{2}}{2}=\frac{(2 v+2)(2 v+1) 2 v}{12} .
$$

It is easy to verify that every ordered triple of distinct elements of $R^{*}$ belongs to at least one directed quadruple of $K^{*}$, which is by (3) sufficient for $\left(R^{*}, K^{*}\right)$ to be a $\operatorname{TQS}(2 v+2)$.

It is straightforward to check that $(R, K)$ is a $\operatorname{DMTS}(2 v+1)$ of the TQS $\left(R^{*}, K^{*}\right)$.

The choice of TQS $(A, B)$ in the above proof was arbitrary, which means that there exists a large number of TQS $(2 v+2)$ having $(R, K)$ as a subsystem.

REMARK. Each MTS $(R, K)$ such that its equivalent M-loop is commutative has the property given in the preceding theorem, but there are numerous examples of MTSs satisfying the conditions of Theorem 7, which have noncommutative equivalent M-loops.

If $(\bar{R}, \cdot)$ is an M-loop, then it is not difficult to prove that the implication $a b=$ $c d \Longrightarrow b a=d c$ is equivalent to $(a b) c=c(b a)$.

An open problem is whether Theorem 7 can be proved without any conditions for the $\operatorname{MTS}(R, K)$.

\section{REFERENCES}

[1] J. Doyen, 'Constructions of disjoint Steiner triple systems', Proc. Amer. Math. Soc. 32 (1972), 409-416.

[2] B. Ganter and H. Werner, 'Co-ordinatizing Steiner systems', in Topics on Steiner systems, Annals of Discrete Mathematics 7, 1980, pp. 3-24.

[3] A. Hartman and K.T. Phelps, 'The spectrum of tetrahedral quadruple systems', Utilitas Math. 37 (1990), 181-188.

[4] C.C. Lindner, 'On the number of disjoint Mendelsohn triple systems', J. Combin. Theory Series $A 30$ (1981), 326-330.

[5] E. Mendelsohn, 'The smallest non derived triple system is simple as a loop', Algebra Universalis 8 (1978), 256-269.

[6] N.S. Mendelsohn, 'A natural generalization of Steiner triple systems', in Computers in number theory (Academic Press, New York, 1971), pp. 323-338.

[7] N.S. Mendelsohn, 'A single groupoid identity for Steiner loops', Aequationes Math. 6 (1971), 228-230.

[8] K.T. Phelps, 'A survey of derived triple systems', in Topics on Steiner systems, Annals of Discrete Mathematics 7, 1980, pp. 105-114. 
[9] R.W. Quackenbush, 'Near boolean algebras I: Combinatorial aspects', Discrete Math. 10 (1974), 301-308.

[10] R.W. Quackenbush, 'Algebraic speculations about Steiner systems', in Topics on Steiner systems, Annals of Discrete Mathematics 7, 1980, pp. 25-35.

[11] R.G. Stanton and N.S. Mendelsohn, 'Some results on ordered quadruple systems', in Proceedings of the Louisiana Conference on Combinatorics, Graph Theory and Computing (Utilitas Math. Publ., 1970), pp. 297-309.

[12] Z. Stojaković, 'Alternating symmetric n-quasigroups', Univ. u Novom Sadu Zb. Rad. Prirod.-Mat. Fak. Ser. Mat. 18 (1983), 259-272.

[13] Z. Stojaković, 'A generalization of Mendelsohn triple systems', Ars Combin. 18 (1984), 131-138.

[14] Z. Stojaković and R. Madaras, 'On teterahedral quadruple systems', Utilitas Math. 29 (1986), 19-26.

Institute of Mathematics

University of Novi Sad

Trg D. Obradovića 4

21000 Novi Sad

Yugoslavia 